\title{
IUPAC Focus on Digital Health
}

\section{by Helle Møller Johannessen and Ulla Magdal Petersen}

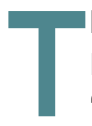

he G-Science Academies of Science and of Medicine published a statement in 2020 titled "Digital Health and the Learning Health System." The statement is interesting and relevant to the work of the Div. VII Chemistry and Human Health, and especially to the Subcommittee on Nomenclature for Properties and Units (SC-NPU).

\section{The G-Science Academies}

The G-Science Academies of Science and of Medicine is a group of national science academies, regularly issuing statements to address some of the world's greatest challenges through the use of science and technology. In 2020, leaders of the national science academies of Brazil, Canada, China, France, Germany, Global Young Academy, India, Indonesia, Italy, Japan, Nigeria, South Korea, the United Kingdom, and the United States signed a statement on "Digital Health and the Learning Health System" (found at https://www.nationalacademies.org/ our-work/joint-science-academies-statements-on-globalissues) that seems especially relevant to the work done in IUPAC. The scope of the document is impressive, describing the many aspects and needs of future Digital Health technology. The statement stresses the need for extensive individual, organizational, national and international collaboration. Some of these aspects have been a concern of IUPAC for decades, especially via IUPAC Division VII, Chemistry and Human Health, and the Division welcomes cooperation with G-Science Academies on these aspects.

\section{Future development of digital technologies}

The development of digital technology is not only ongoing, it is accelerating. There is a need to address uses and risks of digital applications and infrastructure we cannot really imagine today. We (the authors) have for decades been active in various parts of the digitalizing of Danish health care, and while its state today is far beyond what we imagined possible at the start, even the latest and best of health care applications and infrastructure have demonstrated risks and flaws not imagined by the designers. Health care data are very personal, but they are also very valuable in many fields both inside and outside the health care sector. With Health care data being communicated both nationwide and worldwide, it is certain that unplanned access to, recombination of and use of data, and automatic decision making on basis

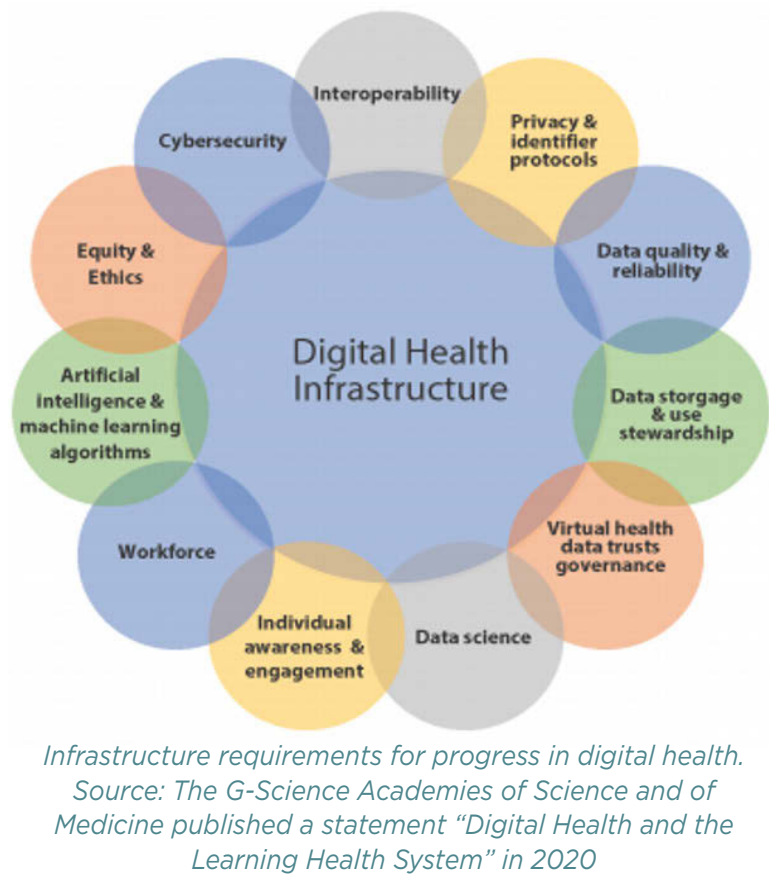

of these data, both in and outside the health care field, will happen. There is a need to evolve not only international technology standards, allowing us to improve the functionality of our tools. We also need ethical and legal standards, economic and political tools, and international agreements for the use of our information. This calls for international participation. But more important, we need to think outside the traditional risk management concepts, because despite all technical improvement over decades, health data can still be lost, inaccessible, misunderstood, misused and stolen.

The G-Science statement recognizes this in the section Data reliability, storage and access:

Structure and maintenance guidelines as well as international cooperation is essential. Equally important are protocols for data storage, access, control, sharing, and use. In principle, authority over individual data lies with the individual from whom they derive, and access to and control of use belongs to the individual or their designee. Every step in the use of information, however, generally requires an element of ceding control, as well as the potential for value to be added. Economic, legal, philosophical and practical issues must be addressed. The differences that exist among nations concerning data access, control, and monetization clearly present limiting circumstances for the contributions of digital health. Thus, mechanisms for ongoing cooperative exchange are needed. 


\section{Relevance to IUPAC Division VII Chemistry and Human Health}

The G-Science statement analyzes and describes the present and future benefits of evolving digital applications and infrastructure, and lists key priorities for future development:

- Cybersecurity, safety, and privacy

- Interoperability

- Availability of reliable data and information

- Secure virtual data repositories

- Integrative analytics and predictive modelling

- Mathematics of learning

- Knowledge representation \& management

- IT literacy, public understanding, and ethics

These priorities are all extremely relevant, and especially interesting for IUPAC Division VII is the third item:

Availability of reliable data \& information: standards and curation protocols for data and information, including tools to track provenance, and improvements in the amounts and quality of open data.

The work in IUPAC Division VII is concerned with subjects of medicinal chemistry and drug discovery, toxicology, explanatory dictionaries, terminology and nomenclature of properties in clinical laboratory science, and advances in immunochemistry. These activities all are relevant in one way or the other to the aspects of Availability of reliable data \& information for the purpose of supporting global standardization.

For more than 50 years IUPAC has cooperated with other International organizations, notably the International Union of Pure and Applied Physics (IUPAP), the International Organization for Standardization (ISO), the International Bureau of Weights and Measures (BIPM), and the International Federation of Clinical Chemistry and Laboratory Medicine (IFCC), to develop standards for metrology in general.

IUPAC Div. VII has been aiming at describing and communicating health relevant information, e.g.

- International agreement on and publication of descriptions and definitions of the communicated information, based on international scientific nomenclature and classifications, to help solve conflicts and misunderstandings due to differences in tradition and language

- Standards of metrology, especially measurement units, and structured and coded reporting of medical data, to make data safely available for clinical use in communication across time and geography, and to allow for reliable reuse of data for research and statistics.
Results of patient examinations make up the main part of communicated data in health care, and most of the data are concerned with the fields of medication, toxicology, human body chemistry and biochemistry. The nationwide or worldwide digital communication of such data supports individual welfare, research and many kinds of statistical use, but also introduces new risks. The data producers (clinician, clinical laboratory, pharmacology dept. ....) are increasingly unable to control where, in which context and for what purpose these data are used. Data are communicated between many different public and private users via systems using different standards, and are recombined and aggregated for many different purposes, both statistical, scientific, and commercial.

Concern for the safety and privacy of personal data has caused the European Union to issue a General Data Protection Regulation (GDPR) in 2018 (found at https:// gdpr-info.eu/), and other nations have expressed similar concerns.

A committee supported by IUPAC and IFCC on 'Nomenclature for Properties and Units' has worked on the issue of definition and identification of personal medical data in a form that can be transmitted between systems and across geography and time, without corruption. The result is the 'NPU Terminology', a set of well-defined and coded 'patient properties' that may be measured or estimated, reported, communicated and reused for many different purposes without loss of meaning.

\section{The NPU terminology}

The NPU terminology is a medical terminology for identification of patient examination results, often based on sample material from the patient. These examinations are often called 'laboratory examinations', even if many of them today are performed locally, in direct contact to the patient, not in a separate laboratory. It covers many different medical fields:

- Clinical allergology

- Clinical chemistry

- Clinical immunology and blood banking

- Clinical microbiology

- Clinical pharmacology

- Molecular biology and genetics

- Reproduction and fertility

- Thrombosis and hemostasis

- Toxicology

The terminology is used in the clinical setting, delivering information about examination results for individual patients. It offers a safe way to communicate 
and compare patient data between different systems and organizations, both locally and on national basis. In the Scandinavian countries (Denmark, Norway and Sweden) the NPU Terminology is managed by National Authorities, translated into the National languages, and used all through the Health Care systems for both ordering of, reporting on and reimbursement for examinations. Transmission of examination results is usually by direct digital communication from medical laboratory systems into local health care systems. The digital examination results are also stored in National Health Care portals, available to both doctors and patients, enabling comparison of results across both time and geography, and allowing for extracts of data for research.

\section{Metrology standards}

The source and definitions for general measurement concepts in the NPU terminology is the 'International vocabulary of metrology - Basic and general concepts and associated terms (VIM)' published by the Joint Committee for Guides in Metrology (JGCM). It ensures International agreement on the meaning of the concepts of the NPU terminology. The International System of Units (SI), commonly known as the metric system, is the international standard for measurement units.

General terminology standards of ISO and the European Committee for Standardization (CEN), and the IUPAC 'Colour books' on Nomenclature have been essential for developing definitions of the specific concepts involved in NPU definitions. In particular important in the field of health terminology and metrology is IUPACs Compendium of Terminology and Nomenclature of Properties in the Clinical Laboratory sciences (The 'Silver Book') [1]. It is produced with the support of both IUPAC and the IFCC (International Federation of Clinical Chemistry and Laboratory Medicine), and makes recommendations on logical standardized nomenclature, symbols, properties, and units in many disciplines of the clinical laboratory sciences. It has been a central source of terms and definitions for health care data.

\section{Units are essential}

Collecting, comparing, storing and reusing measurement results across time, geography and culture is not easy. Many different measurement units are in use in the world, and the units we use shape our general understanding of and relating to magnitudes. Expressing a well-known measured concept using an unfamiliar unit may make it difficult to estimate its meaning. (If you are used to describing body height in centimeters, a value expressed in feet may make it difficult to estimate if a person's height is above or below normal, and vice versa). The difficulty may be handled technically by converting units for presentation and use in in different settings, or socially by agreeing on using the same system of units everywhere.

The NPU terminology is committed to the International System of Units (SI), held by the BIPM.

Incidentally, while this is being written, the BIPM is preparing for the World Metrology Day on 20 May 2021, commemorating the anniversary of the signing of the Metre Convention in 1875. (worldmetrologyday. org/press_release.html). The theme for this year is Measurement for Health!

Not all patient examinations are measurements, some are observations graded on an ordinal scale, or may be nominal classifications (e.g. names of microorganisms identified). But where results are numerical values on a difference or ratio scale, with a measurement unit, the NPU terminology specify either a SI unit or a referenced WHO international unit (IU).

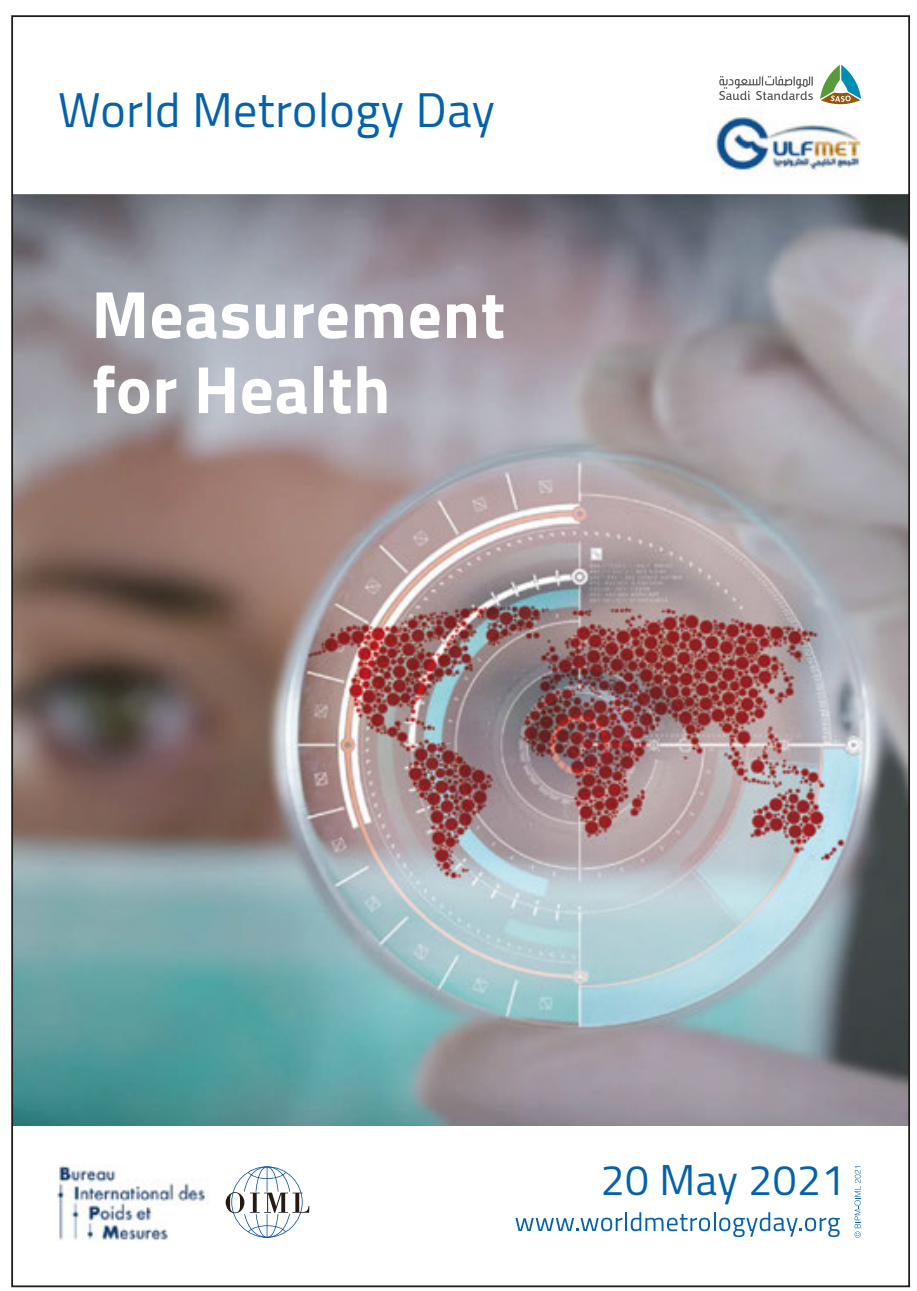




\begin{tabular}{|c|c|c|c|c|c|c|c|c|c|c|c|c|c|c|c|}
\hline & & & & & & \multicolumn{10}{|c|}{2020} \\
\hline & & & & 05.08 & 05.08 & 22.07 & 15.07 & 13.07 & 10.07 & 08.07 & 06.07 & 02.07 & 29.06 & 24.06 & 10.06 \\
\hline & & & Enhed & $08: 43$ & $00: 00$ & $07: 57$ & $08: 48$ & $08: 41$ & 08:44 & $08: 37$ & $08: 57$ & $07: 33$ & $08: 23$ & $08: 30$ & $08: 28$ \\
\hline Folat: $P$ & & a & nmoln & & & 9,5 & & & & & & & & & \\
\hline Hæmoglobin [MCHC]:Er(B) & & a & mmol/L & & & & & & & & & & & & \\
\hline Hæmoglobin; B & a & a & mmo/h & $7,2 \downarrow$ & & $5,9 \downarrow$ & $7,4 \downarrow$ & $6,5 \downarrow$ & $6,6 \downarrow$ & 8,6 & 8,9 & $7,1 \downarrow$ & $7,9 \downarrow$ & $7,1 \downarrow$ & 8,4 \\
\hline Hæmoglobinindhold [MCH]:Ercl... & 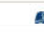 & a & fmol & & & & & & & & & & & & \\
\hline Jern; $;$ & a & a & umoin & & & 18 & & & & & & & & & \\
\hline Leukoblaster; antalk; B & & & $\times 10^{9} /$ & & & & & & & & & & & & \\
\hline Leukocytter; B & a & $a$ & $\times 10^{9} \Omega$ & 6,8 & & $3,4 \downarrow$ & $9,4^{R} \uparrow$ & $12,5 \uparrow$ & $0,66 \downarrow$ & $0,98 \downarrow$ & $14,4 \uparrow$ & $10,4 \uparrow$ & $13,3 \uparrow$ & 5,7 & 8,4 \\
\hline Lymfocytter; B & a & & $\times 10^{9} \Omega$ & 1,9 & & $0,5 \downarrow$ & & $0,9 \downarrow$ & $0,4 \downarrow$ & $0,5 \downarrow$ & $0,6 \downarrow$ & 1,6 & 3,0 & 2,3 & 2,7 \\
\hline Metamyelo.+Myelo.+Promyeloc. & & & $\times 10^{9} / \mathrm{L}$ & & & & & & & & & & & & \\
\hline Metamyelocytter; B & & & $\times 10^{9} \pi$ & & & & 0,1 & & & & & & & & \\
\hline Methæmoglobin; $\mathrm{Hb}(\mathrm{B})$ & & & & & & & & & & & & & & & \\
\hline Monocytter; B & a & & $\times 10^{9} \Omega$ & 0,6 & & 0,2 & & 0,3 & $0,1 \downarrow$ & $0,0 \downarrow$ & $0,1 \downarrow$ & 0,3 & 0,7 & 0,6 & 0,6 \\
\hline Myelocytter; B & & & $\times 10^{9} \pi$ & & & & $0,35 \uparrow$ & & & & & & & & \\
\hline Neutrofilocytter (segmk,+stavk.... & a & $a$ & $\times 10^{9} \pi$ & & & & & & & & & & & & \\
\hline Neutrofilocytter; B & a & a & $\times 10^{9} \pi$ & 3,9 & & 2,5 & & $11 \uparrow$ & $0,1 \downarrow$ & $0,4 \downarrow$ & $14 \uparrow$ & $8,5 \uparrow$ & $9,5 \uparrow$ & 2,5 & 4,7 \\
\hline Promyelocytter; B & & & $\times 10^{9} \pi$ & & & & 0,08 & & & & & & & & \\
\hline Store ufarvede celler; $B$ & & & $\times 10^{9} \Omega$ & 0,11 & & 0,07 & & 0,08 & 0,05 & 0,04 & 0,03 & 0,03 & 0,07 & $0,20 \uparrow$ & $0,20 \uparrow$ \\
\hline Trombocytter; B & a & $a$ & $\times 10^{9} \pi$ & $138 \downarrow$ & & $123 \downarrow$ & $59 \downarrow$ & $53 \downarrow$ & $31 \downarrow$ & $43 \downarrow$ & $100 \downarrow$ & $139 \downarrow$ & 172 & 183 & $118 \downarrow$ \\
\hline Vitamin B12;P & a & a & pmoils & & & 1230 & & & & & & & & & \\
\hline
\end{tabular}

Danish patient overview available on a national platform. By using the unique NPU code and its definition with the unit, the NPU data can be imported directly into the presentation forms. The 'up arrows' and the red colored numbers indicate that the examinations result is above the upper reference limit value. The 'down arrows' and the blue color indicate that the examinations result is below analysis' lower reference limit value. Links to reference intervals and to supplementing information on the investigated property is available as links. Note that the terms are nor the full NPU definitions, but local/national use names.

This makes results reasonably comparable, even though the uncertainty of measured values may differ. If more than one SI unit or international unit are in use for the same patient property, e.g. $\mathrm{mmol} / \mathrm{L}$ and $\mu \mathrm{mol} / \mathrm{L}, \mathrm{NPU}$ uses different identifiers for results with each unit, to avoid misunderstanding.

The result values aim at representing the status of the measurand in the patient at the time of sample collection or direct measurement, not the state of the measurand in the test tube after collection, addition of anticoagulant, centrifugation, or other treatment during the analytical procedure (this is why there are no NPU definitions for blood serum samples-there is no serum in patients. When the blood sample left the patient, it contained blood plasma, and turning it into serum happened in the test tube).

\section{Terminology standards}

All NPU items consist of an identifier (NPUxxxxx) and a formal definition of the examined patient property.

The NPU item definitions are written with a vocabulary of internationally defined concepts and their terms, and references to international definitions of each concept are filed. The NPU definition has three or four parts, describing

- The System-the part of the universe examined. In the medical context usually the patient or a part of the patient, e.g. the blood or skin. Definition of the terms is usually from anatomy sources or other authoritative sources of medical concepts
- The Component-the part of the system that is investigated, e.g. the red blood cells in the blood, or the bacteria on his skin. Definitions are found in relevant international nomenclatures, taxonomies etc.

- The Property examined of the Component in the System, e.g. the number concentration of red blood cells in the blood, or the systematic names (taxa) of bacteria identified in the skin. The definitions of these concepts are found in the IUPAC Color Book series

- A unit-If relevant according to the SI system (e.g. 109/L for the number concentration of red blood cells), or an International Unit (IU) with reference to a specific WHO reference preparation.

An abbreviated form of the NPU definition is used as a name for the NPU item, and users may introduce local or National names in their presentation. The NPU identifier ensures that the complete formal definition can always be found.

\section{Examples:}

Full definitions

NPU01132 Plasma-Albumin; substance concentration $=$ ? micromole per litre

NPU18868 Plasma-Immunoglobulin E; arbitrary substance concentration(WHO 68/341; procedure) $=? \times 10^{3}$ international units per litre

NPU14049 Secretion(Anus)-Parasite(egg); 


\author{
NPU60270 Urine-Mercury; mass concentration = ? \\ microgram per litre
}

Abbreviated descriptions for general use

$$
\begin{array}{ll}
\text { NPU01132 } & \text { P-Albumin; subst.c. }=\text { ? } \mu \mathrm{mol} / \mathrm{L} \\
\text { NPU18868 } & \text { P-Immunoglobulin E; arb.subst.c.(WHO } \\
& \text { 68/341; proc.) }=\text { ? } \times 10^{3} \mathrm{IU} / \mathrm{L} \\
\text { NPU14049 } & \text { Secr(Anus)-Parasite(egg); taxon(proc.) = ? } \\
\text { NPU60270 } & \text { U-Mercury; mass c. }=\text { ? } \mu \mathrm{g} / \mathrm{L}
\end{array}
$$

Terms that may be used in local/National context

$\begin{array}{ll}\text { NPU01132 } & \text { Albumin;P } \\ \text { NPU18868 } & \text { IgE; P } \\ \text { NPU14049 } & \text { Parasite eggs; Anus } \\ \text { NPU60270 } & \text { Mercury;U }\end{array}$

Information about the analytical procedure is generally not part of the NPU definition. But the specification 'procedure' or 'proc.' added to the 'property' part of the definition indicates that results may not be comparable unless the analytical procedure used is the same.

Information about result uncertainty is considered a property of the result value, not of the NPU definition, and is never included in the definition.

With increasing use of the NPU terminology the need for extra information communicated with the examination results has also increased, e.g. on patient condition, sample collection process, methods, instruments, result uncertainty, applied cut-off values etc. It is not feasible to add these very different types of information into the NPU definitions on demand; the definitions would lose their consistency and comparability. But the need for the information is there, and an International standard model and nomenclature for transmitting this kind of supplemental data could be a safe way of handling the problem.

\section{Cooperation with other organizations}

IUPAC Div. VII has always cooperated actively with other organizations in the field of metrology and terminology in Health Care, and continues to do so. IUPAC is a project-based organization, and International projects are the main tool for future development. The NPU terminology continues to evolve and adapt as medical science develops, and the IFCC-IUPAC Subcommittee on Nomenclature, Properties and Units will welcome suggestions for projects in the field.

Cooperation in the field of Availability of reliable data \& information could benefit the work of both the GSA and IUPAC.

\section{Reference}

1. Compendium of Terminology and Nomenclature of Properties Clinical Laboratory Sciences,

Recommendations 2016, IFCC|IUPAC Silver Book, 2nd edition, prepared for publication by G. Férard, R. Dybkaer, X. Fuentes-Arderiu, RSC Publishing, 2017; https://doi.org/10.1039/9781782622451

Helle Møller Johannessen and Ulla Magdal Petersen are both Health Informatics Specialist working at the Danish Health Data Authority in Copenhagen. Helle is currently the Vice President of the IUPAC Chemistry and Human Health Division (Div. VII), and Ulla is Chair of Div.VII SubCommittee on Nomenclature for Properties and Units (SC-NPU). 